\title{
artigo
}

Armada e Silva, H.C.D.; Bernardo da Silva, M.R.; Barros, F.R.S.; Souza, B.R.S.; Medeiros, C.S.; Bernardo da Silva, R.

Análise da mortalidade materna como indicador de gestão do enfermeiro na atenção primária à saúde

\section{Análise da mortalidade materna como indicador de gestão do enfermeiro na atenção primária à saúde}

Analysis of maternal mortality as a management indicator of nurse in primary health care

Análisis de la mortalidad materna como indicador de gestión del enfermero en la atención primaria a lasalud

\section{RESUMO}

Objetivo Refletir acerca dos fatores que contribuem para a mortalidade materna. Metodologia: Tipo descritiva, com abordagem quantitativa e para análise dos dados, foram consultados o Sistema de Informação sobre Mortalidade (SIM), o Módulo de Investigação do SIM e o Sistema de Informação sobre Nascidos Vivos (SINASC) do DATASUS. (SIM), Ciências da Saúde (LILACS). 0 estudo revelou que a Razão de Mortalidade Materna (RMM) no Rio de Janeiro apresentou uma ascendência no ano de 2017 e que a mortalidade continua sendo um aspecto preocupante onde a mesma atinge os países em desenvolvimento, em mulheres de classe econômica menos favorecida. Observa-se a necessidade de assistência pré-natal adequada, equipe multiprofissional qualificada, medidas para impedir a gravidez indesejada, melhora no sistema de referência e contra referência e equidade no atendimento as gestantes.

DESCRITORES: Mortalidade Materna; Complicações na Gravidez; Saúde da Mulher; Estatísticas Vitais.

\section{ABSTRACT}

Objective To reflect on the factors that contribute to maternal mortality. Methodology: Descriptive type, with a quantitative approach and for data analysis, the Mortality Information System (SIM), the SIM Investigation Module and the DATASUS Live Birth Information System (SINASC) were consulted. (SIM), Health Sciences (LILACS). The study revealed that the Maternal Mortality Ratio (RMM) in Rio de Janeiro had an ascendancy in the year 2017 and that mortality remains a worrying aspect where it affects developing countries, in women of less favored economic class. There is a need for adequate prenatal care, a qualified multidisciplinary team, measures to prevent unwanted pregnancies, improvement in the referral system and counter referral and equity in the care of pregnant women.

DESCRIPTORS: Maternal Mortality; Pregnancy Complications; Women's Health; Vital Statistics.

\section{RESUMEN}

Objetivo: Reflexionar sobre los factores que contribuyen a la mortalidad materna. Metodología: Tipo descriptivo, con abordaje cuantitativo y para análisis de los datos, fueron consultados el Sistema de Información sobre Mortalidad (SIM), el Módulo de Investigación del SIM y el Sistema de Información sobre Nacidos Vivos (SINASC) del DATASUS. (SIM), Ciencias de la Salud (LILACS). El estudio reveló que la Razón de Mortalidad Materna (RMM) en Río de Janeiro presentó una ascendencia en el año 2017 y que la mortalidad sigue siendo un aspecto preocupante donde la misma afecta a los países en desarrollo, en mujeres de clase económica menos favorecida. Se observa la necesidad de asistencia prenatal adecuada, equipo multiprofesional calificado, medidas para impedir el embarazo no deseado, mejora en el sistema de referencia y contra referencia y equidad en la atención a las gestantes.

DESCRIPTORES: Mortalidad Materna; Complicaciones em el Embarazo; Salud de la Mujer; Estadísticas Vitales.

RECEBIDO EM: 07/01/2020 APROVADO EM: 07/01/2020

\section{Halene Cristina Dias Armada e Silva}

Doutoranda na UERJ. Mestre em Enfermagem. Coordenador da PAC 5.2. RJ, Brasil. https://orcid.org/0000-0003-0850-8493

\section{Maria Regina Bernardo da Silva}

Mestre em Saúde da Família e Comunidade. MBA em Gestão em Saúde. Docente da Universidade de Castelo Branco. RJ, Brasil. https://orcid.org/0000-0002-3620-3091 


\section{Francisca Reginele de Sousa Barros}

Acadêmica de Enfermagem da Universidade Castelo Branco. RJ, Brasil. https://orcid.org/0000-0002-7963-0501

\section{Daniel Ribeiro Soares de Souza}

Mestre em Saúde e Tecnologia. Docente da Universidade Castelo Branco e Uni São José. RJ, Brasil. https://orcid.org/00000002-9170-6193

\section{Claudia da Silva de Medeiros}

Mestre em Saúde da Família. Docente da Universidade Castelo Branco. RJ, Brasil. https://orcid.org/0000-0003-0471-0731

\section{Raquel Bernardo da Silva}

Mestranda em Atenção Primária à Saúde. Preceptor da Residência de Enfermagem UFRJ. RJ, Brasil. https://orcid.org/00000003-0850-8493

\section{INTRODUÇÃO}

A mortalidade materna é o retrato do número de óbitos ocorridos devido a situações diversas que variam desde o início do período gestacional até um ano de vida do bebê $\hat{e}^{(1)}$. Com essa visão, a Organização Mundial de Saúde (OMS) e a Organização das Nações Unidas (ONU) entraram com medidas de combate para diminuir as incidências, porém, inicialmente não houve significância nos números obtidos ${ }^{(2)}$.

A OMS $^{(3)}$ define a mortalidade materna como o óbito na gestação ou após 42 dias do término da gravidez (puerpério), independente da duração ou o local da gestação, por qualquer causa relacionada ou agravada pela gravidez, ou mediante a conduta relacionada, incluindo fatores acidentais ou incidentais. Dessa forma, identificamos a morte materna, com base em suas causas, definindo-as, como diretas ou indiretas ${ }^{(4)}$.

Morte materna obstétrica direta é aquela resultante de complicações obstétricas da gravidez, parto e/ou puerpério, intervenções, omissões, tratamento incorreto ou cadeia de eventos resultantes das causas mencionadas, por exemplo: hipertensão, hemorragia obstétrica ou complicações da anestesia, são classificadas como morte materna direta ${ }^{(4,5)}$. Morte materna obstétrica indireta é resultante de doenças preexistentes ou que se desenvolvem durante a gravidez. Neste caso, se enquadram, como exemplo, óbito por complicações de doenças cardíacas ou renais ${ }^{(4.6)}$.

Com base nos dados das declarações, o Sistema de Informações sobre Nascidos Vivos (SINASC) consegue apontar para o Ministério da Saúde quais são as prioridades de intervenção relacionadas ao bem-estar da mãe e do bebê, além de fornecer indicadores de saúde sobre pré-natal, assistência ao parto, vitalidade ao nascer, mortalidade infantil e materna ${ }^{(4)}$.

Para cálculo e avaliação epidemiológica, calcula-se a Razão de Mortalidade Materna (RMM), a qual compara o número de mortes obstétricas diretas e indiretas, em relação ao número de nascidos vivos, multiplicado por 100.000 , calculado sob a forma: $\mathrm{RMM}=$ (Número Mortes Maternas / Número Nascidos Vivos) x 100.000.

A questão norteadora: Quais são os fatores que contribuem para a mortalidade materna? E o objetivo do estudo é refletir acerca dos fatores que contribuem para a mortalidade materna. $O$ interesse pela pesquisa deu-se em discutir como as políticas públicas devem ser implantadas para que haja adequado seguimento assistencial visando a redução dos casos de morte materna.

\section{METODOLOGIA}

Este estudo foi do tipo descritivo, com abordagem quantitativa cujo objetivo foi refletir sobre os fatores que contribuem para a mortalidade materna. Para análise dos dados, foram consultados o Sistema de Informação sobre Mortalidade (SIM), o Módulo de Investigação do SIM e o Sistema de Informação sobre Nascidos Vivos (SINASC) do DATASUS. O tempo de coleta, tratamento e análise de dados, combinados à finalização do artigo foi de fevereiro de 2017 a março de 2019. A população deste estudo foram os óbitos ocorridos no município do Rio de Janeiro e devidamente registrados nas bases de dados oficiais. A amostra estudada representou $100 \%$ da população. Por se tratar de dados disponíveis em base de dados de acesso público, o estudo dispensa a submissão ao Comitê de Ética em Pesquisa.

\section{RESULTADOS}

A descrição dos óbitos pareada pelos anos seguiu da seguinte maneira: 61 óbitos em 2015, tais quais subiram para 155 óbitos maternos declarados em 2016. No ano seguinte, em 2017, esse montante elevou-se para 173 e subsequente no ano de 2018 houve uma queda nestes números para $107 \operatorname{casos}^{(4)}$.

A Razão de Mortalidade Materna (RMM) do Município do RJ em 2016 atingiu o índice de 74,7 apresentando elevação no ano de 2017, alcançando 82,9 óbitos/100.000 nascidos vivos. Verificou-se que a área programática como maior RMM foi a Área 5.3. em Santa Cruz e adjacências. Não obstante, esta apresenta um dos piores Índices de Desenvolvimento Social da cidade, caracterizando a dificuldade das mulheres de baixa renda terem acesso aos serviços de saúde de pré-natal, principalmente quando necessário encaminhamento a outro especialista. Em paralelo, as áreas de maior desenvolvimento social (áreas programáticas 2.1 e 2.2 ) apresentam taxas até três vezes menores ao segmento supracitado ${ }^{(7)}$. 


\section{artigo}

Armada e Silva, H.C.D.; Bernardo da Silva, M.R.; Barros, F.R.S.; Souza, B.R.S.; Medeiros, C.S.; Bernardo da Silva, R.

Análise da mortalidade materna como indicador de gestão do enfermeiro na atenção primária à saúde

Conforme o Gráfico 1 abaixo, observa-se de acordo com as áreas programáticas do Rio de Janeiro, a razão da mortalidade materna em 2016 e 2017.

Em 2010, 2015 e 2017 os óbitos obstétricos diretos foram responsáveis por mais de $60 \%$ dos óbitos maternos, sendo sempre a principal causa dos óbitos. Em 2013 e 2014 houve um aumento dos óbitos obstétricos indiretos, porém, em 2015 esses óbitos foram superados, novamente, pelos obstétricos diretos, mantendo-se, então, responsáveis por 30-40\% dos óbitos maternos. Os óbitos classificados como obstétricos não especificados são aqueles em que a investigação não foi suficiente para determinar uma causa direta ou indireta.

As causas classificadas na categoria Aborto (gravidez ectópica, aborto legal) apresentaram perfil decrescente de 2016 para 2017, enquanto as causas classificadas nas categorias de Toxemia Gravídica (Hipertensão, Eclâmpsia e Pré-Eclâmpsia) e Complicações no Puerpério - infecção puerperal) aumentaram nesse mesmo período. Os óbitos por causa indeterminada, transtornos de placenta e AIDS oscilaram entre 2015 e 2017. Causas como Aborto e Toxemia Gravídica podem ser um reflexo da assistência prestada durante o pré-natal no Município do RJ. Conforme o Gráfi-
Gráfico 1. Razão da mortalidade materna por AP. Rio de Janeiro, RJ, Brasil, 2016-2017

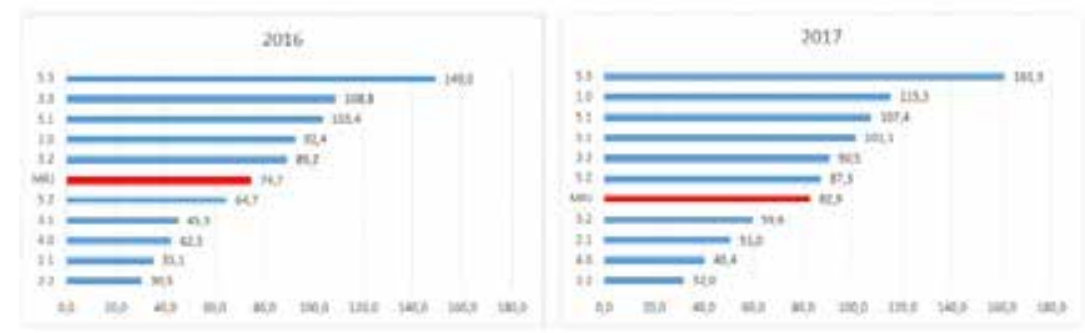

Fonte: SIM. SINASC. SMS-RI. Dados de 2017 suieitos a alteracőes.

Gráfico 2. Distribuição proporcional das causas agrupadas de óbito materno. Rio de Janeiro, RJ, Brasil, 2015-2017

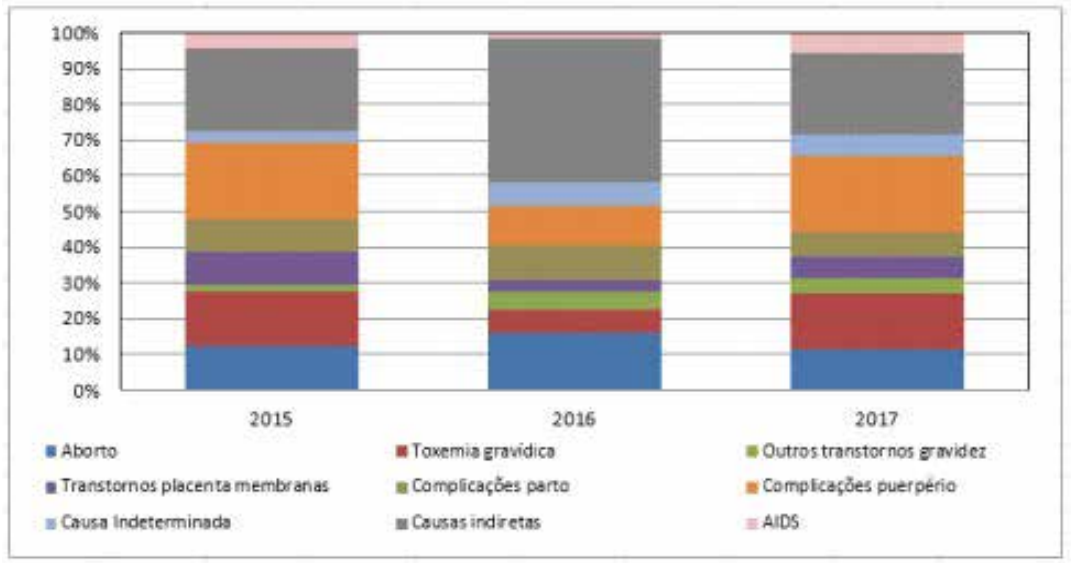

Fonte: SIM, SMS-RJ. Dados de 2017 sujeitos a alterações. co 2 abaixo, observa-se a distribuição por causas agrupadas dos óbitos maternos da secretaria municipal do Rio de Janeiro de 2015 a 2017.

Mesmo parecendo ser uma realidade distante, de acordo com uma pesquisa de âmbito nacional, o pré-natal é uma prática entre as gestantes do país, entretanto algumas incumbências que competem aos serviços deixam a desejar, dentre elas se destacam o número de consultas realizadas e os procedimentos básicos estabelecidos pelo Ministério da Saúde ${ }^{(8)}$.

\section{DISCUSSÃO}

\section{Gestão pública inserida na Mortalida- de Materna}

A gestão do SUS é implantada pelas equipes gestoras que deliberam políticas de saúde, o planejamento é o meio de avaliação que se dá pelo banco de dados e Sistemas de Informação em Saúde - SIS ${ }^{(9)}$.

Com isso, o trabalho do gestor estadual torna-se fundamental no planejamento dos serviços de saúde, sendo este responsável pela coordenação e realização dos planos dos municípios, fornecendo um apoio técnico $^{(10)}$.

Ou seja, um dos papéis da gestão da saúde é a associação, por intermédio de comissões estaduais de integração ensino - serviços (CIES), essa por sua vez é responsável pela formulação, condução e desenvolvimento da política de educação prevista no Artigo 14 da Lei n. ${ }^{\circ} 8080 / 90$ e na NOB/RH - SUS ${ }^{(10)}$.

O Conselho Nacional dos Direitos das Mulheres (CNDM) tornou-se um marco importante na história, por ter conquistado direitos básicos no fortalecimento da democracia $^{(11)}$. A partir Constituição de 1988, as políticas públicas de saúde passam a dar cobertura garantindo a condução de equidade de gênero como a proteção dos direitos humanos das mulheres ${ }^{(11)}$. Além dessas garantias, se faz necessário que o governo tenha ciência da situação que a saúde se encontra, pois, esse parecer torna essencial no quesito de planejar e realizar ações de melhoria para a sociedade em questão ${ }^{(12)}$. 
O objetivo não se isola somente nos descritos acima, a Rede Cegonha traz nos seus objetivos o desejo de potencializar o modelo de atenção à saúde da mulher, seu intuito maior é assegurar o acesso e simplificar o atendimento a este povo ${ }^{(13)}$. Com base nessas informações, algumas medidas foram tomadas para diminuição dos índices de Mortalidade Materna, a OMS investe no fortalecimento de serviços obstétricos, visando a capacitação profissional e contribuindo para redução dos índices de mortalidade ${ }^{(14)}$. A Rede Cegonha foi outra estratégia lançada pelo Ministério da saúde/Secretaria de Vigilância em Saúde (MS/SVS) no ano de 2011, com o intuito de garantir às mães e às crianças o direito ao parto e ao nascimento seguro ${ }^{(15)}$.

Os direitos civis, culturais, socioeconômicos e políticos asseguram deliberadamente o direito de vida livre da Morte Materna, sendo assim, estes obrigam o Estado a obter maneiras concretas para prevenções e realizações de ações que venham a ter impactos concretos, assegurando o primordial, que é a vida ${ }^{(16)}$.

Portanto, o discernimento dos fatores de risco associados à Mortalidade Materna é total relevante para conduzir as ações e intervenções de saúde. Ressaltando ainda a importância do enfermeiro frente às urgências maternas, este se torna protagonista no cuidado individual e coletivo, sendo atribuído das funções do cuidar, que se inicia desde medidas educativas a medidas administrativas $^{(17)}$.

\section{As principais consequências da Mor- talidade Materna}

As principais causas de Mortalidade Materna no Brasil registradas são: hipertensão, hemorragias, infecções pós-parto e abortos $^{(18)}$. De acordo com dados oficiais, o
Brasil se encontra com altos índices de Mortalidade Materna, estes números vão de 50 a 149 por 100.000 nascidos. Contudo, se tratando de referências pouco confiáveis, é nítido que o processo observado diante dos dados é bem superior ao relatado ${ }^{(7)}$.

Mesmo parecendo ser uma realidade distante, de acordo com uma pesquisa de âmbito nacional, o pré-natal é uma prática entre as gestantes do país, entretanto algumas incumbências que competem a esse serviço deixam a desejar, entre elas se destacam o número de consultas realizadas e os procedimentos básicos estabelecidos pelo Ministério da Saúde ${ }^{(8)}$. A dificuldade de submeter a mãe a outro especialista que seja necessário o acompanhamento, principalmente se essa é pertencente ao grupo de baixa renda. Baseado em alguns desses motivos, algumas gestantes procuram a classe médica apenas ao serem acometidas pelas contrações fortes e constantes ${ }^{(7)}$.

A reprodução e sexualidade devem ser protegidas pelo Estado, com a participação efetiva da sociedade. Havendo a necessidade de produção de legislação e políticas públicas visando este cuidado. Quando comparamos óbito materno com essas políticas, percebemos que é preciso trabalhar um desenvolvimento social para combater as desigualdades, constitucionalmente o SUS concede serviços de qualidade, como a ESF, que viabiliza a qualidade necessária em saúde.

A mortalidade materna continua sendo um aspecto preocupante dentro da sociedade brasileira, onde a mesma atinge os países em desenvolvimento, uma realidade em mulheres de classe econômica menos favorecida. Medidas de prevenção e proteção devem ser elaboradas, como por exemplo: assistência pré-natal adequada, equipe multiprofissional qualificada, medidas que impedirão gravidez indesejada, que seja cada vez mais ausente na realidade, vigilância no período puerperal. $\mathrm{O}$ devido preenchimento de atestados de óbitos deve ser feito para identificação de mortes e consequente prevenção dos mesmos. Ainda avaliando, conclui-se que o Município do RJ precisa intensificar os esforços políticos, econômicos e sociais para tentar diminuir o impacto dos óbitos maternos dentro do seu Estado.

É com base nessas políticas de saúde, que as estatísticas de Mortalidade Materna apontam novos rumos para contribuir e enfrentar esse processo ${ }^{(19)}$.

\section{CONCLUSÃO}

É importante organizar um sistema de referência e contra referência efetivo, o intuito é reduzir as desigualdades de acesso ao serviço impostas por diferenças socioeconômicas. Entretanto, dependendo da forma como esta opção for implementada, poderá aumentar a equidade do acesso de usuárias com condições socioeconômicas desfavoráveis, referentes ao acesso de serviços de referência obstétricos. Ao implementar esta opção, é necessária a regulação dos serviços, como: gestão clínica, recursos humanos, condições de acesso aos serviços de saúde e a garantia de transporte adequado no tempo oportuno.

Algumas considerações sobre a promoção da equidade, de acordo com a escolha da opção a ser implantada são necessárias, haja vista a possibilidade de alguns grupos não serem contemplados pelas intervenções. O presente trabalho teve um papel importante no reconhecimento da mortalidade materna, visto que medidas devem ser adotadas para a diminuição significativa do atual quadro.

\section{REFERÊNCIAS}

1. Ferreira EA. Epidemiologia da Mortalidade Materna segundo cor ou raça na região Norte do Brasil, 2006 a 2014. Trabalho de Conclusão de Curso apresentado ao Curso de Graduação em Enfermagem - Universidade Federal
Fluminense [Internet]. Niterói, Brasil 2016 [acesso em 20 fev 2017]. Disponível em: https://app.uff.br/riuff/bitstream/1/3471/1/TCC\%20\%C3\%89dria\%20Aparecida\%20Ferreira.pdf. 


\section{REFERÊNCIAS}

2. Tavares LT, Albergaria TFDS, Guimarães MDAP, Pedreira RBS, Junior EPP. Mortalidade Infantil por causas evitáveis na Bahia, 2000-1012. RECIIS [Internet]. 2016 jul.-set. [acesso em 25 jul 2017]; 10(3). Disponível em: www.reciis.icict.fiocruz.br.

3. Martins EF, Almeida PFBD, Paixão CDO, Bicalho PG, Errico LDSPD. Causas múltiplas de mortalidade materna relacionada ao aborto no Estado de Minas Gerais, Brasil, 2000-2011. Cad. Saúde Pública [Internet]. 2017 [acesso em 27 jul 2017]; 33(1):e00133115. Disponivel em: http://www.scielo.br/pdf/ csp/v33n1/1678-4464-csp-33-01-e00133115.pdf.

4. Resende LV, Rodrigues RN, Fonseca MDC. Mortalidade materna: a violação dos direitos sexuais e reprodutivos e suas consequências. Associação de Estudos Brasileiros Populacionais [Internet]. [acesso em 08 abr 2018]. Disponível em: http://www.abep.org.br/publicacoes/index.php/anais/ article/viewFile/2076/2033. 5. Dias JMG, Oliveira APSD, Cipolotti R, Monteiro BKSM, Pereira RDO. Mortalidade materna. Rev Med Minas Gerais [Internet]. 2015 [acesso em 18 fev 2018]; 25(2):173-179. Disponivel em: http://www.rmmg. org/artigo/detalhes/1771.

6. Mascarenhas PM, Silva GRD, Reis TT, casotti CA, Nery AA. Análise da mortalidade materna. Rev enferm UFPE on-line [Internet]. 2017 nov. [acesso em 19 mai 2018]; 11(Supl. 11):4653-62. Disponivel em: https://periodicos.ufpe.br/revistas/revistaenfermagem/article/view/231206.

7. Pereira LM. Mortalidade materna: como o descaso com a saúde da

mulher impede a igualdade de gênero. Sau. \&Transf. Soc. 2016; 6(1):70-78.

8. Nunes JT, Gomes KRO, Rodrigues MTP, Mascarenhas MDM. Qualidade da assistência pré-natal no Brasil: revisão de artigos publicados de 2005 a 2015. Cad. Saúde Colet [Internet]. 2016 [acesso em 28 mai 2018]; 24(2):252-261 253. Disponivel em: http://www.scielo.br/pdf/cadsc/v24n2/ 1414-462X-cadsc-24-2-252.pdf.

9. Pinheiro ALS, Andrade KTS, Silva DDO, Zacharias FCM, Gomide MFS, Pinto CP. Gestão da Saúde: O uso dos sistemas de informação e o compartilhamento de conhecimento para a tomada de decisão. Texto Contexto Enferm [Internet]. 2016 [acesso em 02 mar 2018]; 25(3):e3440015 Disponível em: http://www.scielo.br/pdf/tce/v25n3/pt_0104-0707-tce-25-03-3440015.pdf.

10. Mendes A, Louvison MCP, Lanni AMZ, Leite MG, Feuerwerker LCM, Tanaka OU, Duarte L, Weiller JAB, Lara NCC, Botelho LDAM, Almeida CAL. O processo de construção da gestão regional da saúde no estado de São Paulo: subsídios para a análise. Saúde Soc. São Paulo. 2015; 24(2):427-437.

11. Gomes AVDM, Silva DRC, Sá MAAFD, Oliveira MDCB, Barboza NA, Mendes PMV. Políticas públicas de atenção à saúde da mulher: uma revisão integrativa. Rev. Interd. Ciên.
Saúde [Internet]. 2017 [acesso em 18 mar 2018]; 4(1):2635. Disponivel em: https://www.google.com.br/search?q=quais+as+politicas+publicas.

12. Lima MM, Aguilar AMM. Análise dos Indicadores de Saúde Materno Infantil de um Município do Estado do Mato Grosso. J Health Sci [Internet]. 2017 [acesso em 01 abr 2018]; 19(2):183-0. Disponível em: http://www.pgsskroton. com.br/seer/index.php/JHealthSci/article/view/4319/367.

13. Oliveira FAMD, Leal GCG, Wolff LDG, Rabelo MX, Poliquesi CB. Reflexões acerca da atuação do enfermeiro na rede cegonha. Rev enferm UFPE on-line. 2016 fev.; 10(Supl.2):86774.

14. Mamede FV, Prudencio PS. Contribuições de programas e políticas públicas para a melhora da saúde materna. Contribuições de programas e políticas públicas para a melhora da saúde materna. Rev Gaúcha Enferm [Internet]. 2015 [acesso em 30 jun 2017]; 36(esp):262-6. Disponivel em: http:// www.scielo.br/pdf/rgenf/v36nspe/0102-6933-rgenf-36-spe-0262.pdf.

15. Santos AKDO, Caveião C. A importância da assistência de enfermagem no puerpério para redução da morbimortalidade materna. Revista Saúde e Desenvolvimento [Internet]. 2014 jul.-dez. [acesso em 01 abr 2018]; 6(3):11. Disponível em: https://www.uninter.com/revistasaude/index.php/saudeDesenvolvimento/article/viewFile/327/220.

16. Galli B, Rocha H, Queiroz J. Relatório sobre Mortalidade Materna no

contexto do processo de implementação da decisão do Comitê CEDAW contra o Estado brasileiro no caso Alyne da Silva Pimentel [Internet]. Plataforma Dhesca Brasil (Curitiba) 2014 [acesso em 05 abr 2018]. Disponível em: http://www. unfpa.org.br/Arquivos/Relatorio_caso_alyne_pimentel.pdf.

17. Reganassi C, Barros KCS, Katch M, Nogueira DP. Mortalidade materna: desafios para enfermagem no enfrentamento da assistência. Revista Fafibe On-Line [Internet]. 2015 [acesso em 10 abr 2018]; 8(1):319-331. Disponivel em: http://unifafibe.com.br/revistasonline/arquivos/revistafafibeonline/sumario/36/30102015190327.pdf.

18. Silva TCD, Varela PLR, Oliveira RRD, Mathias TADF. Morbidade materna grave identificada no Sistema de Informações Hospitalares do Sistema Único de Saúde, no estado do Paraná, 2010. Epidemiol. Serv. Saúde [Internet]. 2016 jul.-set. [acesso em 07 jun 2016]; 25(3):617-628. Disponivel em: https://www.scielosp.org/pdf/ress/2016.v25n3/617628/pt.

19. Viana RDC, Novaes MRCG, Calderon IMP. Mortalidade Materna - uma abordagem atualizada. Com. Ciências Saúde [Internet]. 2014 [acesso em 15 nov 2018]; 22(Sup.1):S141-S152. Disponivel em: http://bvsms.saude.gov.br/bvs/ artigos/mortalidade_materna.pdf. 